\title{
Occurrence of penetrating chest and hearts injury following assaults : a presentation of two cases
}

\begin{abstract}
Thoracic injuries account for $20-25 \%$ of deaths due to trauma. It is known that traumatic cardiac penetration is highly lethal, with case fatality rates of $70-80 \% .1$ With the advent of CT imaging in addition to SXE and ECHO ultrasound scanning, of cardiac injury, an accurate identification of these poten $\neg$ tially lethal injuries have reduced. Such systems of investigations have created a significant improving survival of patients. Most of these patients need surgery and Emergency thoracotomy has an important role in emergency hospitals and can save a lot of lives. Urban areas tend to have higher rates of interpersonal violence and a correspondingly higher percentage of injuries involve penetrating mechanisms compared to rural regions. Delayed pericardial effusion, has rarely been described and it has not been commonly reported in the literature since 1960 but it does happen in patients whose heart penetration has been missed or the patient has had surgery.
\end{abstract}

Keywords: cardiac, emergency, heart, penetrating, trauma

\author{
Volume 8 Issue 2 - 2018
}

\author{
Duncan D Mugala,' Alex Makupe, ${ }^{2}$ Ziddulla, ${ }^{3}$ \\ Dennis Sakala, ${ }^{4}$ Mukosai Simon, ${ }^{5}$ Benaya \\ Chibale, ${ }^{6}$ Charity Sitimela ${ }^{7}$ \\ 'Senior Lecturer, Copperbelt University School of Medicine, \\ Zambia \\ ${ }^{2}$ Senior Suprentendant/ Consultant General Surgeon, Ndola \\ Teaching Hospital, Zambia \\ ${ }^{3}$ Consultant General Surgeon, Ndola Teaching Hospital, Zambia \\ ${ }^{4}$ Consultant Orthpaedic Surgeon, Ndola Teaching Hospital, \\ Zambia \\ ${ }^{5}$ Consultant Urologist Surgeon, Ndola Teaching Hospital, Zambia \\ ${ }^{6}$ Registrar Surgeon, Ndola Teaching Hospital, Zambia \\ 7Intern, General Surgery Ndola Teaching Hospital, Zambia
}

Correspondence: Duncan D Mugala, Ndola Teaching Hospital, 6th Floor Eastwing, Copper Belt University, Zambia, Tel

+260966999957, Email mugaladdcl@yahoo.com

Received: June 28, 2017 | Published: April 26, 2018

\section{Background}

Thoracic injuries account for $20-25 \%$ of deaths due to trauma and contribute to $25-50 \%$ of the general deaths. Approximately 16,000 deaths per year in the United States alone are attributable to chest trauma. ${ }^{1}$ Therefore, thoracic injuries are a contributing factor in up to $75 \%$ of all trauma-related deaths Rohit Shahani et al. ${ }^{1}$ The chest is a very important function of respiration and of protection of the upper abdominal and vital intra-thoracic organs from externally applied force. It is composed of the rigid structure of the rib cage, clavicles, sternum, scapulae, and heavy overlying musculature. Most wounds to these structures can non-operatively be managed. This can be done by simple techniques such as tube thoracotomy. However, the treatment of a stable patient with a normal initial chest radiograph remains controversial in that injuries may be missed. For example, chest wall hemorrhage from the muscular, intercostal, and internal mammary arteries can result in exsanguination and death if the required operative control is missed. So, the primary treatment of chest wall injuries is a combination of pain control, aggressive pulmonary and physical therapy, selective use of intubation plus ventilation, and a close observation for respiratory decompensation. Indications for operative management of the chest wall or sternum injury include the following:

a. Need for thoracotomy for whatever reasons life Heart stabs

b. Laparotomy for Abdominal injuries

c. Large flail segments in patients with borderline premorbid pulmonary status

d. Severe instability and pain and failure to wean from the ventilator after an adequate trial

e. Secondary infections
It must also known that tube Thoracostomy/Thoracotomy which is often a lifesaving procedure and is relatively straightforward, should not be taken too lightly. A review of almost 600 tube thoracostomies revealed a complication rate of $21 \%{ }^{1}$

There is also a known reality that the great vessels of the chest often get injured in times of trauma. They include the aorta, its major branches at the arch (eg, innominate, carotid, subclavian), and the major pulmonary arteries. It must also be kept in mind that the primary venous conduits include the superior and inferior vena cavae and their main tributaries, as well as the pulmonary veins. "Damage to vascular structures depends on the specific location and degree of vessel disruption" says Rohit Shahani et al. ${ }^{1}$ It is kept in mind that arterial injuries are more rapidly fatal. The prevalence of great vessel damages range from $0.3-10 \%$, it has come to knowledge.

It is a known fact that traumatic cardiac penetration is highly lethal, and the case fatality rates ranges from $70 \%$ to $80 \%$. It is known that patients who reach the hospital before cardiac arrest occurs usually survive. Those patients surviving penetrating injury to the heart without coronary or valvular injury can be expected to regain normal cardiac function on long-term follow up. ${ }^{2,3}$

What do we know about Heart injuries?

a. Ventricular injuries are more common than atrial injuries, and the right side is involved more often than the left side.

b. In 1997, the know workers on the heart injuries were Brown and Grover. They noted the following distribution of penetrating cardiac injuries:

- $\quad$ Right ventricle - $43 \%$

- $\quad$ Left ventricle - $34 \%$ 
- $\quad$ Right atrium - $16 \%$

- $\quad$ Left atrium - $7 \%$

c. The Beck triad (ie, high venous pressure, low arterial pressure, muffled heart sounds) is documented in only $10-30 \%$ of patients who have proven tamponade. A large number may be missed.

d. Pericardiocentesis can be both diagnostic and therapeutic, although some centers report a false-negative rate of $80 \%$ and a false-positive rate of $33 \%$. So in diagnosing patients ;it is believed that Ultra sound scanning is much more useful in diagnosing our cardiac injury. The patients can undergo the transthoracic echocardiography (ECHO) and sub-xiphoid exploration (SXE). The ECHO has significant limitations in identifying serious cardiac injuries. But in patients with hemothorax, in hemodynamically stable patients without hemothorax, ECHO picks significant injuries and may be an acceptable diagnostic option for detecting significant cardiac trauma in patients with injuries in proximity to the heart. SXE is better in patients without hemothorax, ${ }^{4}$ Penetrating chest trauma is generally less common but more deadly than blunt chest trauma. The presenting nature of penetrating thoracic trauma can vary widely, from stable patients with few complaints to hemodynamically unstable patients requiring immediate life-saving interventions. Even apparently stable patients with penetrating chest injuries can later deteriorate suddenly. So focused evaluation should be carried out rapidly to assess the life-threatening conditions. Thoracic wall penetration occurs most often from gunshots and stabbings, which comprise up to 10 and 9.5 percent, respectively, of all major trauma in the United States. ${ }^{5}$ In Zambia we do not have this knowledge records to make mention.

Julie Mayglothling et al state that incidences of penetrating thoracic trauma varies geographically. They go on to say that in the United States, 9 percent of all trauma related deaths occur from the thorax injuries, of which one-third involve a penetrating mechanism. In Europe, the incidence of penetrating trauma is reported to be as low as 4 percent. In countries or regions engaged in warfare, up to 95 percent of military deaths may result from a penetrating mechanism. Zambia is not in warfare state, we probably resemble the European incidence but the fact is that we have no records of these facts, not that they don't exist, but that we do not have records. In their review they noted that Urban centers tend to have higher rates of interpersonal violence and a correspondingly higher percentage of injuries involve penetrating mechanisms compared to rural regions. ${ }^{5}$ It is a known fact that approximately 15 to 30 percent of penetrating thoracic injuries require surgery, while in blunt chest trauma patients only less than 10 percent of injuries will need involvement of Surgery.

It is a fact that Cardiac injury due to blunt or penetrating chest trauma is associated with significant morbidity and mortality. Understanding the mechanisms, types, and complications of cardiac injuries and the roles of various imaging modalities in characterizing them is important for appropriate diagnosis and treatment in imaging. These injuries in the past were not well documented, but in the developed countries they are now developed in fast and accurate methods for evaluating the heart and associated mediastinal structures. We too would be happy to carry out a CT scan on all our Chest stabs in Zambia.

In Cardiac Injuries; approximately $25 \%$ of traumatic deaths are caused by cardiac-related injuries with the majority involving either cardiac or great vessels. These may be injuries to the heart due to either blunt force or penetrating trauma to the chest. Most blunt chest injuries are due to motor vehicle collisions (MVCs). We believe this is particularly true in Zambia. This may also be caused by falls particulary in Rural areas in Zambia, explosions, work-related accidents especially Mining industry in Zambia, family related fights and rec $\neg$ reational activities, as well as by cardiopulmonary resuscitation-particularly in the developed countries. In North America, the majorities of penetrating injuries to the heart occur in the civilian environment and are caused by knives or handgun bullets. ${ }^{6}$ In Zambia we need to relook at this and find out what really happens. In Dakar of Senegal the most Penetrating heart injuries causing wounds in the cardiac chambers are due to gunshot or stabbing by knives. Screwdriver is an uncommon weapon but heart injury by screwdriver assault is a rare situation, but it can happen.

In a patient with a foreign body is left in the Chest after a stab, it is better to leave that foreign body impacted into the chest until emergency room can do a thoracotomy for life salvage. ${ }^{11}$ This is because of a known fact that Injury to vital structures, including the heart and great vessels, often leads to rapid death.

Rao R. Ivatury et al state that the early arrival of trained evacuation units at the scene of injury, better supportive therapy while at the site, and rapid transport of victims to designated hospitals had made it a possible chance of an improved survival for heart perforation of heart patients. Their experience also supported the value of immediate emergency room thoracotomy to save the patients. ${ }^{7}$ What do we do when we don't have the system described above and also we don't have the casualty emergency room where thoracotomy can be done immediately is the question we in Zambia are thinking about now.

The increasing frequency of penetrating wounds of the heart is challenging the resources of casualties or emergency care centers in the larger cities not only of the United States of America but in Ndola of Zambia. We Know that Cappelen`s operation is considered to be the first report of a cardiac surgical procedure. Today trauma centers all over the world perform complex cardiac repairs due to penetrating trauma but the mortality is still high- says Kaljusto and Tønnessen. ${ }^{8}$ We are that group of people facing this problem.

A new interesting feature of cardiac penetration is that we have come to know that acute pericardial tamponade after a penetrating cardiac trauma is common. However, delayed pericardial effusion, has rarely been described and it has not been commonly reported in the literature since 1960. In South Africa it has been reported. The most common clinical findings are distended neck veins, dyspnoea, pleural effusion and other features of right heart failure. ${ }^{9}$ In these patients pericardiocentesis is a common treatment for stable patients presenting with acute cardiac tamponade following a stab. These patients are known to develop septic pericarditis. Patients with septic pericarditis should be followed up for the possible development of constrictive pericarditis at a later stage, or could be initially drained by left thoracotomy with pericardiectomy. The diagnosis of a penetrating cardiac patient may be missed in a stable patient, This has been reported in South Africa, the patients present with delayed pericardial effusions and tamponade and the most common causes are Staphylococcus aureus and/or beta-haemolytic streptococcus. ${ }^{9}$

We present two patients who were admitted to the Ndola teaching hospital with Heart penetration stab wounds and were saved. 


\section{Case I}

Our first patient was L M, he was a male 31 years old. The above named came to Ndola teaching hospital on 5th April 2017 at about 22:00 hours with a history of being stabbed around 21:00hours by a known person, using a knife. LM and his friend bought chicken (feet $\&$ intestines) before they left for work so that they could eat when they get back from work. Around 18 hours when they came back from work, they found that the chicken feet had been stolen by one of their friends.

When they confronted the man who had stolen their chicken, he seemed unapologetic so they decided to beat him up and went elsewhere to look for something to eat. After some time had passed the man they had beaten returned with a knife and attacked LM, he stabbed him in the chest and abdomen. The neighboring women came to save LM and tied him up in a cotton cloth commonly known in Zambia as the "Chitenge cloth" and he was rushed to the hospital.

\section{Admission at the hospital}

On arrivals at the Ndola casualty, the patent's airway was normal. He was breathing spontaneously and there was no respiratory distress. There was equal air entry in his chest. His Blood Pressure was 100/70 $\mathrm{mm}$ of $\mathrm{Hg}$. His Pulse Rate was 100 beats per minute. The breathing respiratory rate was 18 breaths per minute. The patient was fully conscience.

On exposure, he had stab wounds on the left anterior chest. He also had a stab wound in the right hypochondrium with omentum protruding out through the wound. Two large bore cannulae were inserted. An urgent cross-match was done and a Full Blood Count (FBC) was collected. Intravenous fluid (IVF) was commenced The patient was booked for the Operation Theatre(OT). The following were carried out and done: The ATT $0.5 \mathrm{ml}$ Intra Muscularly were given. Intravenous drugs of Metronidazole $500 \mathrm{mg}$ and Cefotaxime 1 gram were given. As a follow up the were to be given three drugs per day Intravenously. This was commenced.

The patient was wheeled to theatre and an urgent thoracotomy and laparatomy were performed.

The Thoracotomy and Laparotomy procedures were done. Intraoperative findings were as follows: The patient had a collapsed left lung, There was a penetrating wound in the pericardium in the right heart ventricle. The Sternum was ripped apart and the pericardial tamponade was found and Pericardial opening had to be done. The Penetrated hears was shooting the heart blood out. The stabbed heart was sutured. The abdominal stub wound revealed a tear in the stomach and a linear laceration on the left lobe of the liver. These were repaired.

\section{Investigations and Treatments done:}

The Patient was admitted to the Intensive Care Unit of the Ndola Teachinh Hospital for 24 hours and he was in a stable state through his stay. Because of being stable, he was transferred to the Males ward. After three days he developed a wound infection along his Thoracotomy wound but his Laparotomy was normal. By the tenth day the infection settled down and the patient was discharged out of the hospital, however when he was reviewd on the third day week he was found with a Pneumonia and a Pericardial effusion. These eventually subsided (Figure 1-8).

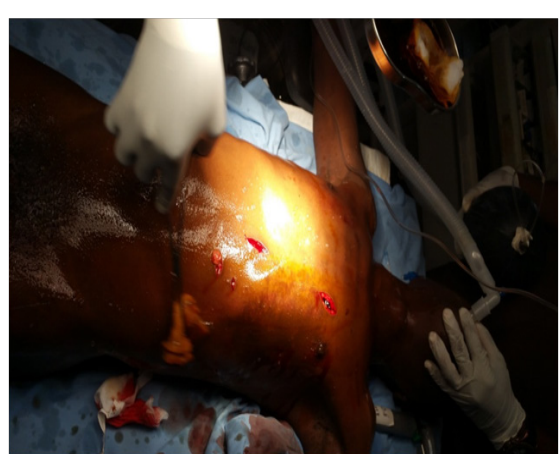

Figure I Multiple abdominal and chest stab wounds.

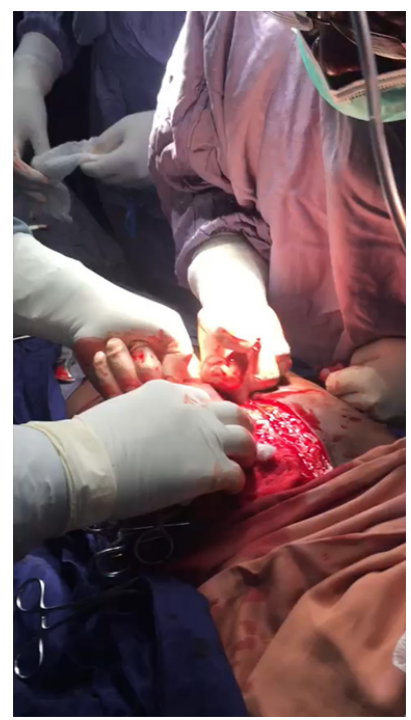

Figure 2 Median sternotomy

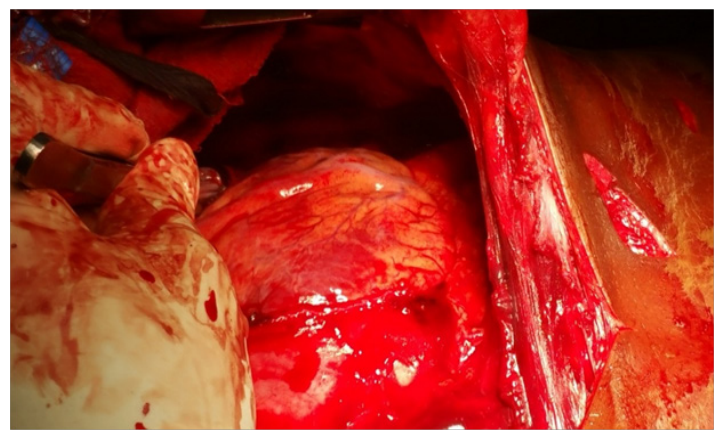

Figure 3 Stabbed heart.

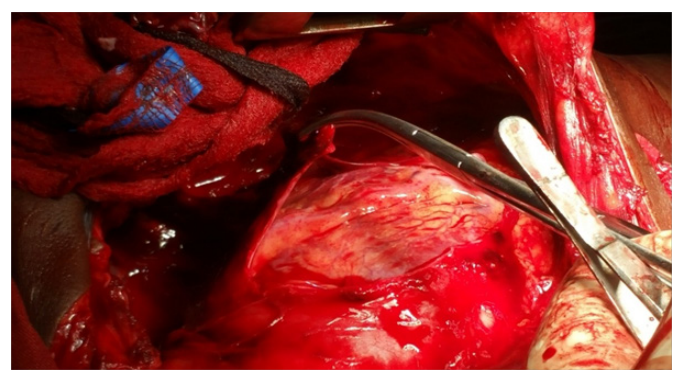

Figure 4 Pericardium opened. 


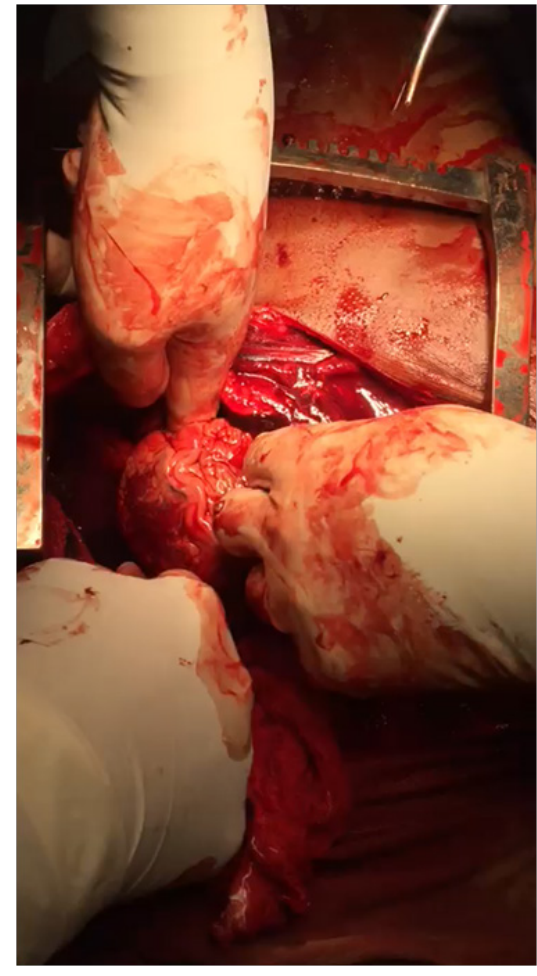

Figure 5 Identifying stab on heart.

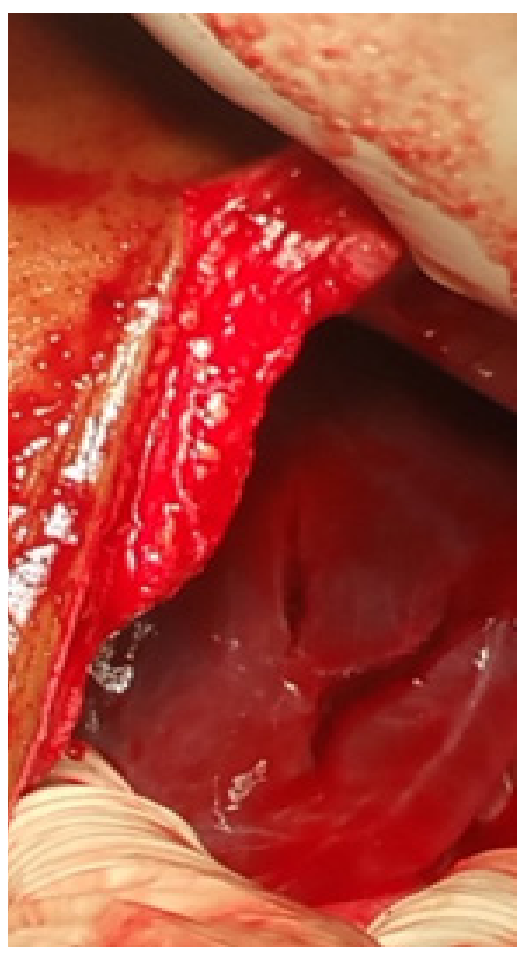

Figure 6 Laparotomy laceration of the liver.

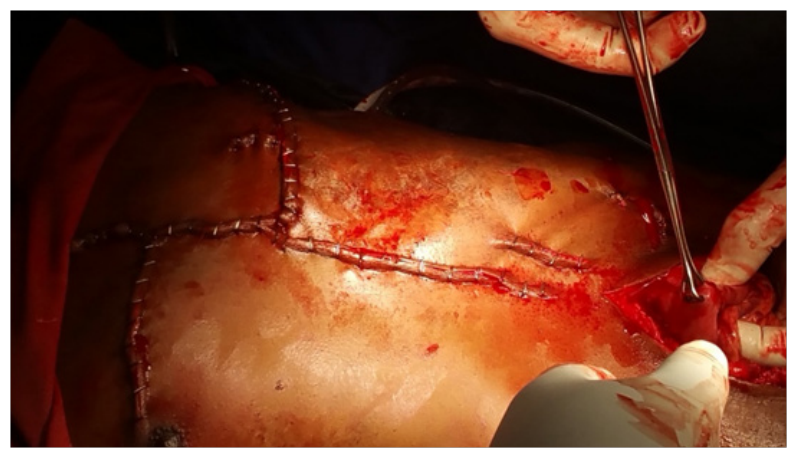

Figure 7 Thoraco- abdominal surgery done and closed

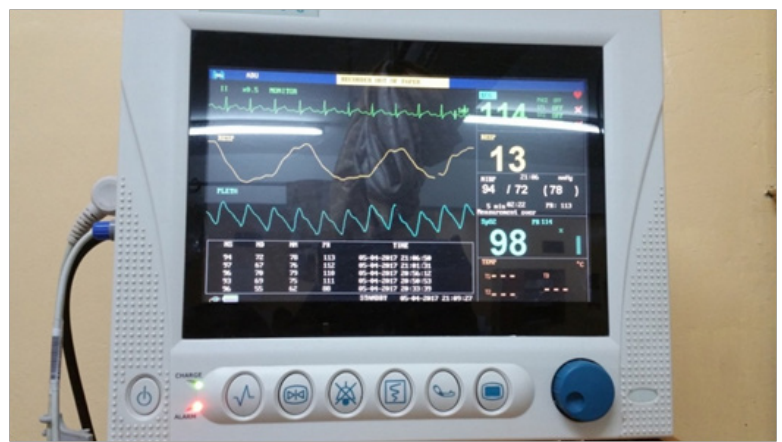

Figure 8 The Patients ICU was Functioning Normally.

\section{Case 2}

Our second patient was M M, a male age 25 years old. He came to Ndola Teaching Hospital casualty on 20th April 2017 at about 03:00hrs with a stab wound on the left anterior chest wall. History obtained from the patient's wife on the day of presentation at NTH was that M M was stabbed by his girlfriend. She said she just found him home bleeding from the stab wound. She didn't give any further report. Latter we obtained more clear detailed from MM on 4th May 2017. The details was that he was stabbed by his wife using a knife. He had a conflict with his wife about 5 days prior to being stabbed. The reasons given for the conflict is that he one day hit his wife with a cooking stick after having a quarrel over her habits of cooking late, taking alcohol and coming home late. This conflict led his wife to leaving home and carrying most of their house property to her mother's house, leaving him only with a mattress. In the evening of the 19th April 2017 he went to take a bit of alcohol at a bar and there he met his "former" wife who was also drinking alcohol. At around 21:00hrs he decided to go home and left his"former" at the bar.

Later that night around midnight he heard a knock on his door only to hear his wife shouting for him to open the door. He opened the door for her and she demanded to collect the mattress. MM was resistant on giving her the mattress and as they were arguing, she picked a knife and aimed to stab him. They struggling with the knife, but his wife stabbed him to the chest.

After being stabbed, MM run to the neighbor's door. The neighbors 
on getting to know what had happened told his wife to take him to the hospital and present to NTH that MM was stabbed by his girlfriend and say that she just found him home bleeding from the stab wound.

MM later denied allegations made that his girlfriend, rather than his wife had stabbed him. He however, did not deny the fact that he has a girlfriend who has his 2 year old child.

\section{Social history}

The patient went up to grade 6 . He was the last borne in a family of six. He takes takes alcohol. He does not smoke. He works as a bus conductor. He has been married for two years with no children from his wife

\section{Admission at the hospital}

On arrivals at the Ndola casualty, the patent's airway was normal He was breathing spontaneously but his respiratory rate was 22 breaths per minute. The Chest was not moving symmetrically. There was Pneumothorax on percussion on the left side of the chest. On Auscultation there was Cardiac Tamponade (distant cardiac sounds). His Blood Pressure was $60 / 40 \mathrm{~mm} \mathrm{Hg}$.His Pulse Rate was 116 beats per minute. The Glasgow coma scale was $15 / 15$. The Pupils were equal and reacting to light. There were no focal neurological signs. There was a laceration on the anterior left side of the chest along the mid clavicular line, about $2 \mathrm{~cm}$ medial to the nipple.

\section{Interventions}

A Size 16 cannular was inserted in the $2^{\text {nd }}$ intercostals space along the midclavicular line. Air rushed out and the patients breathing was normalised. Two large bore cannulae were inserted and the Intra Vascular Fluid (IVF) was commenced. An urgent Cross-match and Full Blood Count was collected. A Chest Drain(CD) was inserted. Immediately more than $250 \mathrm{mil}$ of blood was drained. Because of this, the $\mathrm{CD}$ was clamped. The Consultant Surgeon was called out to come and attend to the patient. In the mean time the following things were done ATT O.5 mililres Intra Muscular were given .Metronidazole $500 \mathrm{mg}$ and Cefotaxime 1 gram were Intra Venously commenced. The patient was wheeled to theatre for an urgent thoracotomy Intraoperative findings were. There was leaking out of the heart each time it contracted. This was because the ventricle left side of the heart was stabbed, it had cut about $1 \mathrm{~cm}$ in size. A $2 / 0$ vycril suture on the rounded needle was used (Figures 9-12).

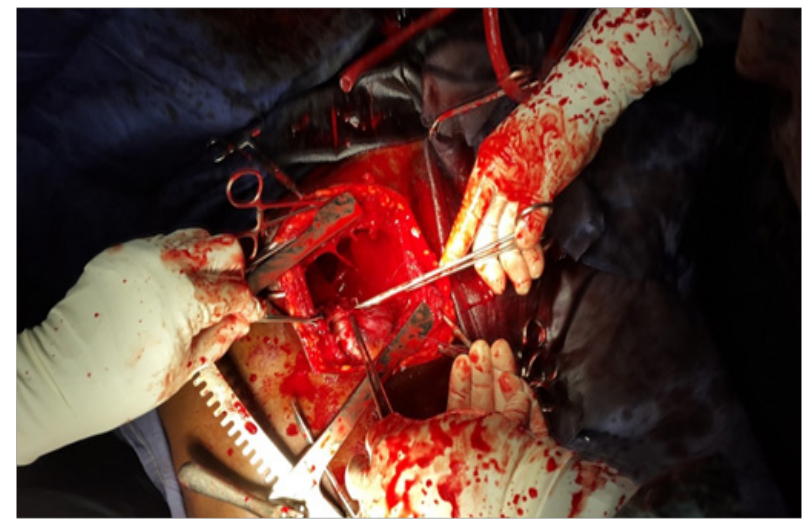

Figure 9 The Heart being sutured

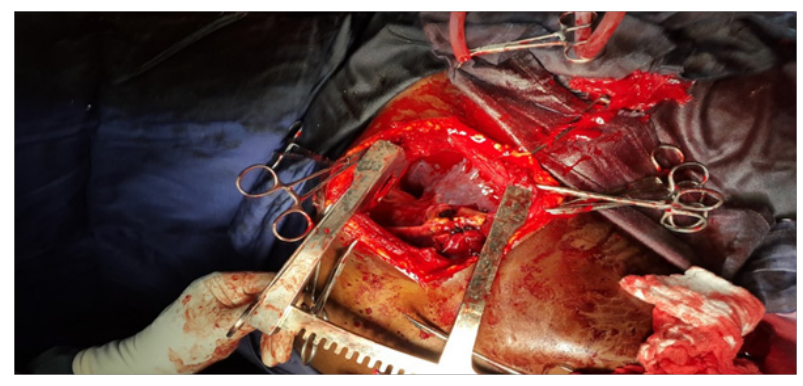

Figure 10 The Heart sutured.

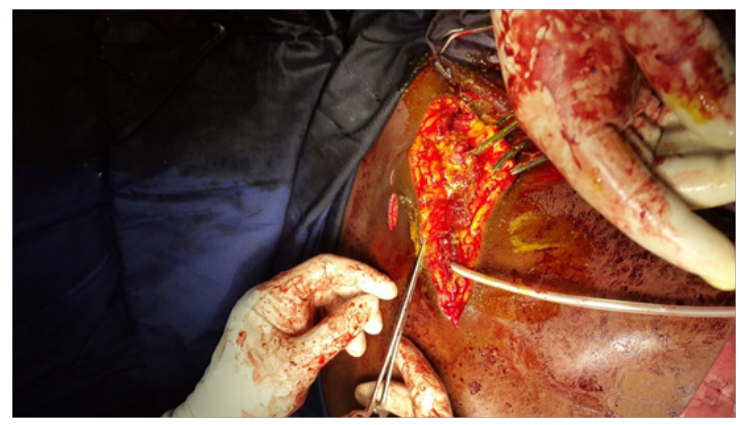

Figure I I The skin being closed

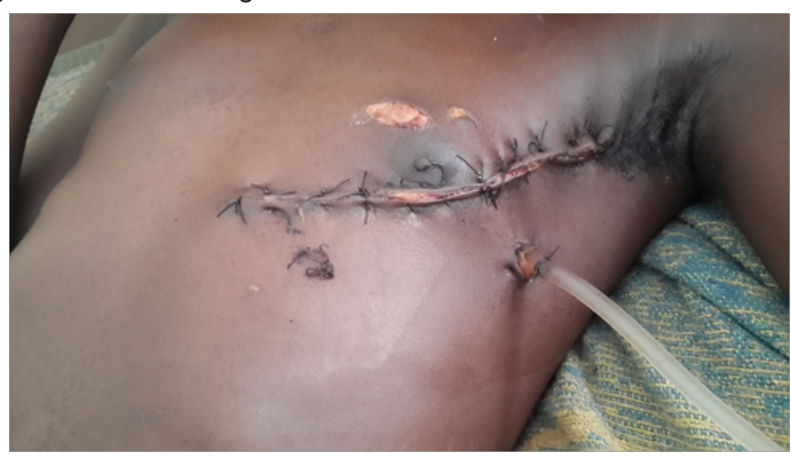

Figure 12 Three days post Operation.

\section{Investigations and treatments done}

The Patient was admitted to the Intensive Care Unit of the Ndola Teaching Hospital for 24 hours and was in stable state through his stay. Because of this stable state, he was transferred to the Males ward. He has settled down very well and we hope he will sort out his social state

\section{Discussion}

From the 1970s, trauma networks have saved thousands of lives in the hospital setting. However, few recent works have been done to evaluate the patients who die in the field or on their way to the hospital. Davis JS, Satahoo et al report that the leading cause of death is neurotrauma, followed by hemorrhage, asphyxia, and combined neurotrauma and hemorrhage. Mostly these were injuries from hemorrhage and chest injuries. ${ }^{2}$ Both our two cases were cases of hemorrhage and chest injuries that could have led to death but were saved.

Heart penetration from chest stabs are not very common. In fact Mariadason JG et al. studied a case of 324 patients admitted to Harlem Hospital Center from July 1981 to June 1986 with stab 
wounds of the thoraco-abdominal region. Their aim was to determine the incidence of trans-diaphragmatic penetration from the thoracoabdominal trauma. They had no Heart perforation at all. ${ }^{3}$ Of our two patients, one was admitted with a thoraco-abdominal trauma. He had no trans-diaphragmatic penetration but he had a heart perforation. It is Known that these two patients we had were supposed to undergo the Pericardio-centesis and Ultra sound scanning which is much more useful in diagnosing our cardiac injury. The ECHO Scanning has significant limitations in identifying serious cardiac injuries in patients with haemothorax. However in hemodynamically stable patients without haemothorax, ECHO does not misses significant injuries and is an acceptable diagnostic option for detecting significant cardiac trauma in patients with injuries in heart injuries. In patients with haemothorax, the SXE ultra sound scanning gives a better report. ${ }^{4}$ At our hospital we have no history of carrying out the patient heart scanning examinations because we have not seen these patients at frequent times. In a period more than five years we have seen only two patients. So, ultra sound scanning on all our penetration hearts patients is rare. However, it must be said that if imaging modalities are available, the value of multi-detector computed tomography (CT) for the initial evaluation of patients with blunt or penetrating chest trauma, are highly essential: With the advent of CT imaging of cardiac injury has brought in an accurate identification of these rare but potentially lethal injuries. It has become significant for improving survival. Unfortunately we were not in that state of development but we now have acquired a CT imaging equipment. We hope we will be using this modern Imaging equipment and will save more lives. Our two patients came into the Ndola Teaching Hospital just a week apart and our CT was going to be of great value.

It has also taught us that we must be aware that there are cases of patient who die outside the hospital. Davis JS, Satahoo et al. ${ }^{2}$ have reported that in their review it included trauma deaths in 2011. This was that some patients were not transported to a hospital they had died at the scene or dead on arrival. ${ }^{2}$ We have no idea how many of our patients actually die outside the hospital. A study must be carried out.

It is known that penetrating chest trauma is generally less common but more deadly than blunt chest trauma. Julie Mayglothling et al. have done a retrospective study and have come up with the facts that, chest injuries are a relatively common cause of preventable death among trauma patients. This was because they carried out small retrospective reviews. In their view; thoracic wall penetration occurs most often from gunshots and stabbings, which comprise up to 10 and 9.5 percent, respectively, of all major trauma in the United States. ${ }^{5} \mathrm{We}$ have seen only two patients in Six years. Our cases seem rare. Here we have no clear record of what happens in our community. There may be patients who die before the come to our attention. The two cases we had were both stabbings. Julie Mayglothling et al. ${ }^{5}$ also state that incidences of penetrating thoracic trauma varies geographically. They said that in the United States, 9 percent of all trauma related deaths occur from injuries to the thorax, of which one-third involve a penetrating mechanism. In Europe, the incidence of penetrating trauma is reported to be as low as 4 percent. However, in countries or regions engaged in warfare, up to 95 percent of military deaths may result from a weapon penetrating mechanism. Zambia is not in warfare state, we probably resemble the European incidence but the fact is that we have no records of these facts. They go on to state that in their view the Urban areas tend to have higher rates of interpersonal violence and a correspondingly higher percentage of injuries involve penetrating mechanisms compared to rural regions. ${ }^{5}$ Our two patients were from Ndola which is a City area in Zambia. We can say that perhaps their mind is likely to what we experienced also.

Steven J. et all state that in Cardiac Injuries; approximately 25\% of traumatic deaths are caused by cardiac-related injuries with the majority involving either cardiac or great vessels. These may be injuries to the heart due to either blunt force or penetrating trauma to the chest. ${ }^{6}$ We have only had two cases so we really cannot state our position.

Rao R. Ivatury et al describe the facts that Cardiac injuries of patients with penetrating were treated at Lincoln Medical and Mental Health Center in America and their opinion was that: They had total of 75 patients with penetrating cardiac from 1974 to 1980.They had wenty-two patients $(29.3 \%)$ who were unconscious on arrival and had no detectable vital signs, cardiac activity, or spontaneous respirations. In their study they noted that; the early arrival of trained evacuation units at the scene of injury, the better supportive therapy, and the rapid transport of victims to designated hospitals had contributed a high improved survival of patients. Their experience clearly supports the value of immediate emergency room thoracotomy to save the patients. ${ }^{7}$ In our cases we used the main theatre. Our casualty room does not have emergency theatre room. Rao R. Ivatury et al. ${ }^{7}$ performed immediate resuscitation of their patients and employed intercostal or sternal splitting incisions.

In their theatre emergency room, they prevented arrested hearts and permitted relief of tamponade. In our two patients the following actions happened; one had sternal splitting incision and the second had intercostal incision, We are beginning to see these type of patients who are calling us to rethink our situation. We are not the only ones. Kaljusto and Tønnessen report the report case of a young man who suffered a large stab wound (SW) in the left ventricle and left atrium in addition to a lung injury for approximately 2 hours before undergoing operative surgery. ${ }^{8}$ Our Second patient underwent Cardiac surgery within four hours.

We know that acute pericardial tamponade after a penetrating cardiac trauma is common. However, delayed pericardial effusion, has rarely been described and it has not been commonly reported in the literature since 1960, pericardiocentesis was and is a common treatment for stable patients presenting with acute cardiac tamponade following a stab. David G harris et al. ${ }^{9}$ demonstrated a case of pericardial effusion over a period of an 8-year period, They had 24 patients who were diagnosed with delayed pericardial effusions following a recent stab wound over the chest. It was carried out from January 1994 to December 2001. The most common clinical findings are distended neck veins, dyspnoea, pleural effusion and other features of right heart failure. We had a patient who had pleural effusion and Pericardial effusion, he was our first patient following surgical operation. It appears that in South Africa they have had patients with heart penetrations and have not been operated on but they survived only to develop pericardial effusion. ${ }^{9}$ This being interesting, we feel missing a penetrative heart patient may result in death. One surgical case is what Ordog GJ et al found out: Of the four among their patients with initially unsuspected cardiac injuries, two patients died in the operating room. These were Asymptomatic patients with normal findings on chest $\mathrm{x}$-ray films. All patients should have close outpatient follow-up, these are the patients who develop pleural or Pericardial effusion. ${ }^{9-12}$ 
Cardiac injuries analysis is highly important: Cardiac injury has traditionally been considered to be a positive prognostic factor, compared to findings of non-cardiac thoracic or abdominal injuries. A meta-analysis by Rhee, et al. determined that survival rates were highest for isolated penetrating cardiac injuries (19\%) compared to penetrating non-cardiac thoracic (11\%)-This is particularly so if the greater Vessels are the major damages. ${ }^{6}$ The penetrating abdominal were $4 \%$, and multiple penetrating injuries were $<1 \%$. Overall survival rates were $17 \%$ after stab wounds and $4 \%$ after gunshot wounds. Sameh Ibrahim Sersar and Mohammed Adel Alanwar recommend and emphasize the importance of emergency medicine education programs on rapid diagnosis of traumatic injuries with early intervention, and adequate hemodynamic and respiratory support. They go on to say that emergency thoracotomy has an important role in emergency big volume hospitals and can save a lot of lives. ${ }^{11}$ Our two patients were Cardiac penetration patients and fortunately the grater vessels were missed. We followed the recommend facts of the Advanced Trauma life support (ATLS) guidelines and emergency thoracotomy in penetrating thoracic injuries based on their rules. ${ }^{11}$

One typical reason is what Ordog GJ et al. ${ }^{12}$ found out: Of the four among their patients with initially unsuspected cardiac injuries, two patients died in the operating room. These were Asymptomatic patients with normal findings on chest x-ray films. All patients should have close outpatient follow-up, these are the patients who develop pleural or Pericardial effusion..$^{9,12}$

\section{Conclusion}

At Ndola in Zambia a city town we saw our knife heart stabs which occurred at about the same month for the first town in six years. May be most of our heart stab wounds victims die before they arrive but it is a warning that this may be on the rise as our city is becoming a big city on the Copperbelt of Zambia. We must plan to use most available investigations like SXE and ECHO ultra sound scanning and the CT on these patients and by and large be ready to perform thoracotomy to save the patients. One of our two patients developed pleural and Pericardial effusion which is a reminder that these patients with heart penetration injuries may develop pleural and Pericardial effusion, the most common causes are Staphylococcus aureus and beta-haemolytic streptococcus. Aggressive antibiotics will save their live. However some cases of missing penetrative heart injuries may be discovered dead.

\section{Acknowledgements}

None.

\section{Conflict of interest}

The author declares there is no conflict of interest.

\section{References}

1. Rohit Shahani, Jeffrey C Milliken. Penetrating Chest Trauma Treatment \& Management. 2015.

2. Davis JS, Satahoo SS, Butler FK, et al. An analysis of prehospital deaths: Who can we save? J Trauma Acute Care Surg. 2014;77(2):213-8.

3. Mariadason JG, Parsa MH, Ayuyao A, et al. Management of stab wounds to the thoracoabdominal region. A clinical approach. Ann Surg. 1988;207(3):335-40.

4. Meyer DM, Jessen ME, Grayburn PA. Use of echocardiography to detect occult cardiac injury after penetrating thoracic trauma: a prospective study. J Trauma. 1995;39(5):902-7.

5. Julie Mayglothling, Eric Legome, Maria E Moreira, et al. Initial evaluation and management of penetrating thoracic trauma in adults. 2017.

6. Steven J Co, Charlotte J, Yong-Hing, et al. Role of Imaging in Penetrating and Blunt Traumatic Injury Trauma/Emergency Radiology. 2011.

7. Rao R Ivatury, Pravin M Shah, Katsuki Ito, et al. Emergency Room Thoracotomy for the Resuscitation of Patients with "Fatal" Penetrating Injuries of the Heart. The Annals of Thoracic Surgery. 1981;32(4):377-385.

8. Kaljusto ML, Tønnessen T. How to mend a broken heart: a major stab wound of the left ventricle. World Journal of Emergency Surg. 2012;7(1):17.

9. David G Harris, Jacques T Janson, Jacques Van Wyk, et al. Delayed pericardial effusion following stab wounds to the chest. Eur J Cardiothorac Surg. 2003;23(4):473-476.

10. Dien PA, Diop MS, Ciss AG, et al. Penetrating Heart Injury due to Screwdriver Assault: A Case Reports. Service de Chirurgie Cardiovasculaire et Thoracique, CHUN Fann, Dakar, Senegal in Cardiology. 2015.

11. Sersar SI, Al, Anwar MA. Emergency thoracotomies: Two center study. $J$ Emerg Trauma Shock. 2013;6(1):11-5.

12. Ordog GJ, Wasserberger J, Balasubramanium S, et al. Asymptomatic stab wounds of the chest. J Trauma. 1994;36(5):680-4. 